\title{
DIE NATURWISSENSCHAFTEN
}

\section{Entwicklung und Stand der Forschung über die Röntgenstrahlen.}

\author{
Von Prof. Dr. H. Sieveking, Karlsruhe.
}

Die eminente praktische Bedeutung der Röntgenschen Futdeckung gab der Technik den Anlaß, sich der weiteren Entwicklung dieses neuen Gebietes (ier Physik anzunehmen. Der Einblick in das Innere des Organismus, die Erfüllung des alten Traumes, daß dem Arzte der Zukunft der menschliche Köorper? durchsichtig wie ein Gebilde aus Glas erscheinen wirde, lassen auch den ganz ferustehenden Laion die ungeheure Tragweite der Entdeckung erkennen. Die Entwicklung der Funkeninduktoren, die neue Technik der an Größe und Durchdringungskraft immer wachsenden Röhren, haben ganze Industrien neu ins Leben gerufen.

Dem trägt die Literatur über diesen Gegenstand natürlich Rechnung. So gibt es eine Reihe guter Bücher über Röntgentechnik. Die eigentliche physikalische Seite der neuen. Strahlen ist dadurch otwas in den Hintergrund gedrängt worden. Es entm sprach daher sicherlich einem dringenden Bedürfnis, als aus berufener Feder ein Buch über die Physik der Röntgenstrahlen angekündigt wurde, das über die Fortschritte auf diesem Gebiet berichten sollte.

Fs liegt jetzt vor als Band 45 der Monographiensammlung "Die Wissenschaft", Verlag von Fr. Vieweg \& Sohn. Der Verfasser, Herr Privatdozent Dr. R. Pohl, Berlin, ist aus mancherlei Grïnden für diese Aufgabe prädestiniert. Wir wollen hier nur kurz darauf hinweisen, daß er zusammen mit Professor Walter (Hamburg), der auch für die technische Entwicklung der Röntgenstrahlen bahmbrechend gewirkt hat, in den Streit über die Beugung der Strahlen eingegriffen hat und hier aufklärend tätig war, daß er ferner die heiBumstrittene Frage nach der Richtigkeit der Geschwindigkeitsbestimmung durch Marx zusammen mit Franck in ein neues Stadium gebracht hat.

Die ursprüngliche Absicht des Schreibers dieser Zeilen, über dieses Pohlsche Buch zu referieren, hätte der Wichtigkeit dieser Publikation wohl nicht genügend Rechnung getragen. So soll im Einverständnis mit und auf Wunsch der Leitung dieser Zeitschrift der Rahmen eines Referates überschritten werden, und in gekürzter Form ein Uberblick über den jetzigen Stand des neuen Zweiges der Physik gegeben werden. Wir werden uns dabei eng an das Pohlsche Buch halten, und hoffen, das wohlverdiente Interesse für dasselbe gebührend zu steigern. Ebenso wie der Verfasser dürfen auch wir hier die Technik der Strahlen als bekannt voraussetzen. Man hat ja auf Kongressen fast jedes Jahr Gelegenheit, die wunderbaren Fortschritte auf dem Gebiet des Induktorium- und Röhrenbaus zu bewundern. Mam weiß, daß Aufnahmen von Bruchteilen von Selkunden genügen, um sogar bewegte Organe in den verschiedenen Stadien und Phasen zu verfolgen.
Die physikalische Seite der Röntgenstrahlen ist gerade in letzter Zeit durch die Entdeckung von Laue und seinen Mitarbeitern in ein ganz neues Stadium getreten. Dieser Punkt, der in dem Pohlschen Buch nur noch als Anhang behandelt werden konnte, und über den die Diskussion noch nicht abgeschlossen ist, rückt die bis dahin in vielen Punkten clunkle Strahlung in eil ganz neues Licht, insofern die ersten gana unbestrittenen Interferenzen einer. sicheren Schlub auf den wellenförmigen Charakter zulassen. Daß weit über die engere Bedeutung fur die Röntgenstrahlen hinaus die Lauesche Entdeckung uns einen Einblick in das Innere der Kristalle gestattet, sei hier nur erwähnt und später ausführlich besprochen.

Die Entstehung von Röntgenstrahlen ist an eine plötzliche Geschwindigkeitsänderung von Elektronen geknüptt. Wird ein Vakuumrohr genügend weit evakuiert, so geht von allen Teilen, die von der vom negativen Pol ausgehenden Kathodenstrahlung getroffen werden, eine neue charakteristische Strahlung aus. Bei den ersten klassischen Versuchen von Röntgen diente die Glaswand als Quelle der neuen Strahlung; bei den vervollkommmeten Röhren bedient man sich einer metallischen, "Antikathode". Die wichtigsten Eigenschaften der ,neuen Art von Strahlen" sind alle von. Röntgen selbst in seinen Originalmitteilungen beschrieben. Die Strahlen erregen die photographische Platte, den Leuchtschirm und andere fluoreszierende Stoffe, sie ionisieren die Luft, sie durchdringen auf Grund der versehiedenen Absorbierbarkeit die inhomogenen Hindernisse unter Schattenbildung. Die von einer Antikathode ausgehende Strahlung ist nicht homogen. Schon die erzeugenden Kathodenstrahlen sind je nach der Axt der Frzeugung mehr oder weniger inhomogen.

Wemn die bewegten Elektronen auf ein Hindernis stoßen, so werden sie nicht sofort ihre ganze Geschwindigkeit verlieren, sondern sie dringen in das Atomgefiuge der Antikathode ein und werden dort auf den verschiedensten Bahnen zickzackförmig vordringen, bis endlich ihre Geschwindigkeit null geworden ist. So resultiert eine regellos verteilte Emission. Dazu gesellt sich eine Sekundärstrahlung, die die Verhältnisse noch verwickelter gestaltet. Durch geeignete Filter läBt sich die inhomogene Strahlung. von der geringen Reststrahlung homogener Art trennen. Es bleiben dann wenige Prozente einer homogenen oder gerichteten Strahlung übrig.

Die bei Elektronenstrahlung angezeigte Analyse durch ein Magnetfeld lieferte bei der neuen Strahlung ein durchaus negatives Resultat. Die genauesten Versuche hierüber hat Walter angestellt. Ein Feld von 19000 Gauß ergab keine Ablenkung. Dies ist ein (Grund dafür, daß die R-Strahlen 1 ) häufig mit den $\gamma$-Strahlen des Radiums in Beziehung gebracht

1) Trm weiteren Verlauf gebrauchte Abkürzung fux Röntgenstrahlen. 
werden. Auch der Nachweis ciner Brechung verlief resultatlos. Faben wix es mit einer Wellenstrahlung zu tun, so ist jedenfalls die Wellenlänge eine sehr kleine, woraus folgt, dal der Brechungsexponent sich nur wenig von eins unterscheiden kann. Die Versuche, eine Beugung der R-Strahlen nachzuweisen, bilden den Gegenstand einer Reihe von Arbeiten, von denen in erster Linie die Versuche von Haga und Wind, dam die von Walter und Pohl Erwahnung verdienen. Die positiven Ergebnisse der ersteren führten die letzteren auf eine optische Täuschung zurück. Die einwandfreiere Ausmessung der Photogramme nach dem Verfahren von Koch schaltet solche Fehler aus; doch lieBen sich auch hiermit keine ganz sicheren Belege für eine Beugung finden. Sie ist zwar bei den Aufnahmen von Walter und Pohl nicht unwahrscheinlich, doch müssen dabei zwei Bedingungen erfüllt sein: es darf bei der sechsstündigen Exposition der Träger der Apparatur nicht gewackelt haben oder sich durchgebogen, noch larf eine erhebliche Diffusion des Bildes in der mpfindlichen Schicht der Platte aufgetreten sein. Theoretisch hat Sommerfeld die Beugung der R-Strahlen behandelt. Die Verhältnisse liegen anders als bei gewöhnlichem, sicher periodischem Licht. Es ist keine seitliche Abweichung der Lin ien absolut gleicher Intensität zu erwarten, sondern ur ein Abrücken derselben von der Mittellinie. Die Verhältuisse bei don. Walter-Pohlsehen Aufnahmen stehen in Einklang mit der Thoorie, sind aber den oben genamnten Einschränkungen unterworfen.

Auf diesem Wege war man also nicht zu einer Bestimmung der GröBe, die sowohl für eine periodische Strahlung als Wellenlänge wie für einen Impuls als Breite desselben charakteristisch ist, gelangt. Solange der periodische Charakter der R-Strahlung nicht nachgewiesen war, kounte man nur ron einer Impulsbreite sprechen. Als Ergebnis der Waltex-Pohlschen Photogramme ergibt sich als oberer Grcnzwert $=4 \cdot 10^{-9} \mathrm{~cm}$.

Die allererste Deutung der R-Strahlen als longitudinale Strahlung ist nicht von Bestand gewesen. In der Richtung des Bremsweges, in der nur cine longitudinale Strahlung auftreten könnte, ist die Strahlung gleich null.

Die Polarisation ist naturgemäB sehr schwer nachzuweisen, da die Strahlung, wie schon erwähnt, inhomogen ist, und nux wenige Prozonte die Bedingung einer gerichteten Strahlung crfiullen. Die Abhängigkeit der Impulsbreite vom Lmissionswinkel wurde mit Sicherheit von Fyedrichs nachgewiesen, die Polarisation mit Sicherheit dureh Barkla. Statt der photographischen Intensitätsmessung dient die elektrometrische, bei welcher die erhöhte Leitfähigkeit der Luft in einer Ionisationskammer der Messung zugrunde liegt. Späterc Messungen, in erster Linie die von Baßter, bestätigen das Frgebnis, daB die R-Strahlung polarisiert ist. Die Ebene der maximalen Intensität der Emission ist senkrecht zur Bahn der erzeugenden Kathodenstrahlen. Mit wachsender Entladungsspannung wird diese Bevorzugung weniger deutlich, da dann der Anteil der inhomogenen
Strảhlung zunimmt, und dic Verhältuisse weviger eiowandfrei liegen.

Dic Beobachtungen von Bafler boten der theoretischen Behandlung durch Sommerfeld das nötige Material zur Nachprüfung der von ihm berecheten Energieverteilung. Auch die Erscheinung, dab hinter der Antikathode eine Strahlung auftritt, läßt sich durch die Theorie erklären.

Die wichtige Größe der Impulsbreite ist zuerst ron Wien auf theoretischem Wege abgeleitet worden, und zwar liegt der Ableitung eine von Abraham stammende Gleichung für die Emissionsenergie bei der Bremsung zugrunde. Ist die Impulsbreite $\lambda=c \tau$, und ist die Energie gegeben als Funktion ron Elektronenladung, Verzögerung, Lichtgeschwindigkeit, und endlich Verhältio der Geschwindigkeit des Elektrons zur Lichtgeschwindigkeit, ist ferner der Nutzeffekt der R-Strahlung in Prozenten der Kathodenstrahlung bekannt, so läßt sich $\lambda$ berechnen unter Zugrundelegung der klassischen Werte für $e / m$ und $e$. Man erhält so für $\lambda$ den Wert $1,15 \cdot 10^{-10} \mathrm{~cm}$.

Auf einem anderen. Wege hat Wien und ebenso Stark aus einer ron Einstein stammenden Gleichung, nach welcher die kinetische Energie eines durch Schwingungen von der Frequenz y ausgelösten Elektrons nur gleich $h \cdot \nu$ oder gleich einem ganzen Vielfachen dieser Gröbe sein kam, die Impulsbreite berechnet. Plancl hat bekanntlich die neue Hypothese von der quantenhaft erfolgenden Energieemission in die Theorie der Strahlung eingefuhrt. Der Grundgedanke ist der, daß die Emissionsenergie gestaffelt sei, d. h. aus nicht weiter unterteilbaren Quanten bestehe. Die Größe des Planckschen Wirkungsquantums ist aus Strahlungsmessungen bekannt.

Ist

$$
\frac{h}{x}=h \nu=\frac{1}{2} m r_{0}^{2}=\varepsilon \mathrm{Y}
$$

so ist, $\operatorname{la} \frac{1}{\tau}=\frac{r}{\lambda}$ ist,

$$
\lambda=300 \frac{h c}{* V}=1,26 \cdot \frac{10^{-4}}{V} ;
$$

da num h nach Planck gleich $6,55 \cdot 10^{-27}$ erg/sck. ist, so ergibt sich für harte Strahlen mit $V=60000$ Volt

$$
\dot{\lambda}=2 \cdot 10^{-9} \mathrm{~cm} \text {. }
$$

Dieser Wert ist in guter Ubereinstimmung mit dem. oberen Grenzwert aus den Beugungsversuchen vou Walter und Pohl. Freilich divergiert er stark von. dem anderen Wert, der sich aus dem Nutzeffekt ableiten lieB. Nach Sommerfeld aber läBt sich diese Diskrepanz so erklären, dab der Nutzeffekt zu groß angenommen war. Er muß in dem Verhältnis der gerichteten zu der ungerichteten Strahlung reduziert werden. Es ergibt sich durch die Rechnung $5,4 \%$ für das Verhältmis $E_{R} / E_{K}$, was wiederum mit den experimentellen Ergebnissen vou Bapler gut in Einklang steht. Die universelle Bedeutung des Planckschen Wirkungsquantums läBt also auch hier, ebenso wie bei der Deutung des photoelektrischen und des Haber-Justschen 
Reaktionseffekts eine Anwendung zu, die fern von der ursprünglichen Domäne, der Thermodynamik liegt, ein Beweis für die grobe Fruchtbarkeit dieses Gedankens. Nach Poincaré ist, die Quantenhypothese die kühnste Idee der Physik seit Newtons Tagen.

Wir betrachten weitere Eigenschaften der R-Strahlen:

Wie schon Röntgen festgestellt hat, exregen R-Strahlen, die auf irgend ein Medium treffen, dieses in der Art, dal eine neue Strahlung von ihm ausgeht. Sagnac hat sie sekundäre R-Strahlung genannt. Nach Barkla, Sadler u. a. setzt sich diese Strahlung aus zwei Phänomenen zusammen; einer Art Zerstreuung der primären Strahlen, und daneben einer zweiten, stark von der Natur des durchstrahlten Mediums abhängigen Strahlung. Dividiert man die relative Intensität der senkrecht zur Primärstrahlung zerstreuten Sekundärstrahlung durch die Dichte des Gases, in dem der Versuch ausgeführt wird, so ergibt sich, solange das Atomgewicht des Gases unter 32 bleibt, mit Ausnahme des Wasserstoffes, für 12 verschiedene Gase der gleiche Wert. Gleiche Massen von Elementen $(A<32)$ emittieren also relativ gleiche Selundärstrahlung. Es deutet dies auf eine Art Verwandtschaft \%wischen der Strahlung und dem Bau des Atoms. Vielleicht mag die Hoffuung, mittels R-Strahlen das Gefinge des Atombaus in der Art eines radioaktiven Vorgangs zu lockern, hierdurch bestärkt verden. Freilich bedurfen die Aufsehen erregenden Mitteilungen von neueren Umwandlungen, die Ramsay mittels R-Strahlen erzielt haben will, noch der Bestätigung.

Der Zerstreungsfaktox läBt sich in Beziehung bringen zu der schon mehrfach erwähnten Energie cer Primärstrahlung. Aus dem gemessenen Wert liaßt sich dann ableiten, wieviel Elektronen in jedem einzelnen Atom der zerstreuenden Substanz vorhanden sein müssen. Es ergeben sich $2 / 3 A$ Elektronen, wenn $A$ das Atomgewicht der Substanz ist. Diese Zahl ist für das Verständnis vom Bau der Atome von fundamentaler Bedeutung. Die Frage nach der Zusammensetzung der Atome aus materiellen Bestandteilen und Elektronen hat bekanntlich durch die Berechnungex von Nicholson, über die vor kurzem Fajans in dieser Zeitschrift ausführlich berichtet hat, grobe Bedeutung gewonnen, insofern die Uebereinstimmung zwischen den be rechneten und den bestbekamnten Atomgewichten zahlreicher Elemente mit kleinem Atomgewicht eine Aufsehen erregend gute ist.

Eine weitere Eigentümlichkeit der R-Strahlen haben Barkla und Sadler entdeckt. AuBer dem Streungsvermögen besitzen die Elemente die Fähigkeit, eine Sekundärstrahlung zu emittieren, dexen durch ihre Absorbierbarkeit definierte Impulsbreite einen für jedes Element ganz charakteristischen Wert besitzt. Djese Strahlung ist vollständig homogen, und ihre Absorption folgt eincm cinfachen Exponentialgesetz:

$$
J=J_{0} \cdot e^{-\mu x} \text {. }
$$

So ist bereits für eine grobe Anzahl von Grundstoffen eine typische Absorptionskonstantro gemessen worden, die ebenso charakteristisch für die betreffende Substanz ist wie beispielsweise eine bestinmte Spektrallinie.

Auch eine selektive Absorption hat sich nachveisen lassen; doch lag hier bisher die grobe Sohwierigkeit vor, daB die. Impulsbreite nicht mit der gleichen Schärfe bekannt war wie die Wellenlinge in der Optik. Das Maximum der Durch lässigkeit beim Eisen z. B. liegt bei einer Impulsbreite, der eine Kathodenstrahlengesehwindigkeit von etwa $5,6 \cdot 10^{9} \mathrm{~cm}$ entspricht. Der Zusammenhang dex charakteristischen Strahlung mit der selektiven Absorption ist ein Analogon zur Optik, wo ebenfalls das Auftreten einer Fluorescenzstrahlung an das einer selektiven Absorption gebunden ist.

Noch deutlicher aber ist die Analogie zwischen R-Strahlen und Licht auf dem Gebiet der Elektronenemission durch beide. Wie Dorn gefunden lat, ist die Absorption von R-Strahlen verknüpft mit einer Emission von Kathodenstrahlen. Nach Bestelmeyer ist für letztere e/m $=1,72 \cdot 10^{7}$ ein Wert, der sich wenig von dem Standardwert 1,76 $10^{7}$ unterscheidet. Die Messung erfolgt durch magnetische Ablenkung. Die so gemessene Geschwindigkeit ergibt sich als unabhängig von der Intensität der erzeugenden R-Strahlen, was bekunntlich beim Licht seit langem erwiesen ist. Die Zahl der Elektronen ist der Intensität der R-Strahlen proportional und ebenso der bolometrisch gemessenen Energie derselben.

Einige weitere Figenschaften der Röntgenstrahlung mögen noch kurz angefuhrt werden. Die Kondensation übersättigten Wasserdampfes an Kernen, die durch R-Strahlen erzengt werden, ist durch die klassischen Versuche von $J$. J. Thomson zur Bestimmung des Elementarquantums be kannt geworden. Wenn man sich auch jetzt lieber der Radiumpräparate zur Bildung von Tonen bedient, so ist doch die Erzeugung durch R-Strahlen keineswegs ganz zur Seite gedrängt. Die Sichtbarmachung der Elektronenbahnen durch Wilson gibt einen reizwollen Einblick in die Mikrowelt. Auch feste und flüssige Dielektrika werden durch $R$ Strahlen ionisiert.

Der photoelektrische Effekt der R-Strahlen gab Marx ein Mittel an die Hand, die Ausbreitungsgeschwindigkeit derselben zu messen mit dem Ergebnis, dab sich R-Strahlen mit genau gleicher Geschwindigkeit fortpflanzen wie die elektrischen Wellen an Drähten, d. h. mit Liehtgeschwindigkeit. Wenn Poht und Franck auch an der Richtigkeit dieses Resultats keine Zweifel hegen, so glauben sio doch, dab die Meßmethole nicht einwandfrei ist. Die Diskussion hieriber ist noch nicht abgeschlossen. Es wäre zu bedauern, wenn die scharfsinnigen und eleganten Messungen ron Herrn Mar: $x$ auf nicht einwandfreior Basis aufgebaut wären. Da der Streit noch nicht beendet ist; so sej hier nicht weiter darauf eingegangen.

Die Versuche von Blondlot, die Geschwindigkeit zu messen, haben kein einwandfreies Resultat gehabt. 
Von chemischeu Wirkungen der R-Strahlen ist in erster Linie die Wirkung auf die photographische Platte zu nennen; ferner die Wirkung auf Glas, das sich färbt und blau wird, ebenso wie unter dem Einfluß der Becquerelstrahlung. Man führt dies zurück auf eine Manganausscheidung. Die physiologischen Wirkungen intensiver $\mathrm{R}$-Strahlung sind leider früher unterschätzt worden. So sind öfters sehr schwere Verbrennungen, die sogar Amputationen notwendig machten, beobachtet worden. Die Dosierung der Strahlung ist schwierig. Sorgfaltiges Abblenden dureh Bleischirme ist dringend geboten.

Was wir in dem Pohlschen Buch vermissen, ist cin Hinweis auf die interessanten Versuche von Jensen über Münzabbildungen durch R-Strahlen. Sehr merkwürdig ist die Abbildung beider Prägungen auf der Photographie. Diese düfte ihre endguiltige Erklärung gefunden haben (s. Photograph. Jahrb. 1909, p. 55 u, ff). Dagegen bedarf es nach den Mitteilungen Jensens noch genauerer Untersuchungen darüber, welcher Natur die Strahlen sind, die - und zwar vor allem bei den zur Silber- und Platingruppe gehörigen Metallen beim Negativ die den Vertiefungen entsprechenden hellen und den Erhabenheiten entsprechenden dunklen Stellen geben. So viel konnte allerdings einwandfrei gezeigt werden, daß diese ,Umkehrerscheinung" mittelbar herbeigefuhrt wird durch eine sekundäre Röntgenstrahlung, welche rom Boden der hölzemen Kasette ausgeht, in dem die Photographische Platte mit den darauf befindlichen Münzen bzw. Medaillen oder sonstigen Metallen liegt, und welche damn, von der Rückseite her, das Glas der photographischen Platte durchdringend, die der Schichtseite anliegende Seite der angewandten Metallgegenstände trifft.

Fin hervorragender Fortschritt in der Physik der R-Strahlen datiert von der Entdeckung Laues und seiner Mitarbeiter, denen es gelang, eine Interferenz der R-Strahlen nachzuweisen. Damit eine Interferenz an gitterartigen Gebilden. zustande kommt, darf die Gitterkonstante nicht wesentlich größer sein als die Wellenlänge der zur Interferenz gelangenden Strahlen. Nach obigen Darlegungen exgab sich für die Impulsbreite oder unter Voraussetzung regelmäliger Strahlung für die Wellenlänge der Wert $10^{-9}$. Der Abstand zweier Molekülzentren ist von der Größenordnung 10-8. So lag der Gedanke nahe, die Molekularstruktur als Beugungsgitter zu benutzen. Diese Idee ist von Laue verwirklicht worden, Das Raumgitter eines Kristalles, das dem Auge nicht, sichtbar ist, aber seine Existenz durch den kristallinischen anisotropen Charaliter vermuten läbt, kann mach Laue zum Ausgangszentrum eimer Sekundärstrahlung gemacht werden, die auf der photographischen Platte Interfereubilder von grober Sehärfe und wunderbarer Schönheit liefert. Welch ein Reiz, in das Innere der Moleküle hineinzuschauen und zum ersten Male diese doch immer hypothetischen Bausteine der Materie sichtbar zu machen! Welch genialex Gedanke, das gewöhnliche Licht, dessen zarte Schwingungen sich als zu grob erwiesen, zu ersetzen durch das neue Licht mit einer Wellenlänge $10^{-9} \mathrm{~cm}$ ! Und welche Erweiterung der Wellenskala! Um 13 Oktaven wird das Spektrum exweitert. Das sichtbare Licht umfaßt. etwa cine Oktave; das neue, nicht direkt wahrnehmbare, aber mit einfachen Hilfsmitteln erkenntliche Licht eroffnet ganz ungeahnte Perspektiven. Wemn es gelingt, mit seiner Hilfe in das Innere der Körper einzudringen, so mag noch. manches Rätsel gelöst werden. Es bietet jeden falls eine große Befriedigung, daß die Moleküle, iiber deren Zahl und Gewicht etwa 25 Methoden mit gleichem Ergebnis AufschluB gaben, dem zweifelnden Auge näher gebracht worden sind. Die Wellenlänge berechnet sich aus den Interferenzversuchen von Laue $7 u 1,27-4,83 \cdot 10^{-9} \mathrm{~cm}$.

Wir haben uns in unserer Darstellung eng an das Pohlsche Buch gehalten. Freilich haben wir damit die Inhaltsangabe keineswegs erschöpft. Wir raten jedem, den das Thema interessiert, die Lektüre des Buches dringend an. Es ist sehr klar und fesselud geschrieben, und, obwohl der Verfasser: an manchen Ergebnissen persönlich beteiligt ist, tritt er doch aus dem Rahmen objektiver Darstellung liaum heraus.

Sollte es uns gelungen sein, für dies wichtige Gebiet der Physik einiges Interesse zu wecken, so wäre der Hauptzweck dieser Zusammenfassung. erreicht.

\section{Der Schwindel und seine Beziehungen zum Bogengangapparat des inneren Ohres. Bogengangapparat u. Kleinhirn. (Historische Darstellung. Eigene Untersuchungen.)}

\author{
Von Unit.-Doz, Dr. Robert Bárány, Wien.
}

Dreht man sich mehrmals rasch um seine Achse und bleibt dann plötzlich stehen, so empfindet man Schwindel, d. h. die Umgebung scheint sich um einen zu drehen und schlieBt man die Augen, so empfindet man eine scheinbare Drehung des eigenen Körpers. Dies ist der Drehschwindel, von dem hier die Rede sein soll. Auch die Empfindung, die man verspürt, wenn man von einem hohen Turm in die Tiefe schaut, wird als Schwindel bezeichnet, aber bei diesem „Höhenschwindel" dreht sich nichts. Der sei aus unserer Betrachtung ausgeschaltet; er ist wahrscheinlich nur psychisch bedingt. Der Drehschwindel war schon den Griechen des Altertums bekannt und wird sehr gut von ihnen beschrieben. $\operatorname{Im} 16$, 17. und 18. Jahrhundert konnte ich eine grobe Menge von Dissertationen über den Sehwindel anffinden. Fs sind mehr als 100. Das Figentümliche ist, daß sie alle nahezu dasselbe enthalten. Man hatte sich im Mittelalter fast vollständig das Beobachten abgewöhnt. Wer ein Buch schrieb, der studierte die Schriften seiner Vorgänger und schrieb schlieBlich mit kleinen Anderungen das ab, was vor ihm gesagt worden war. So kommt es z. B., daß in der uns interessierenden Frage des Drehschwindels von verschiedenen Autoren darüber spekuliert wird, ob 